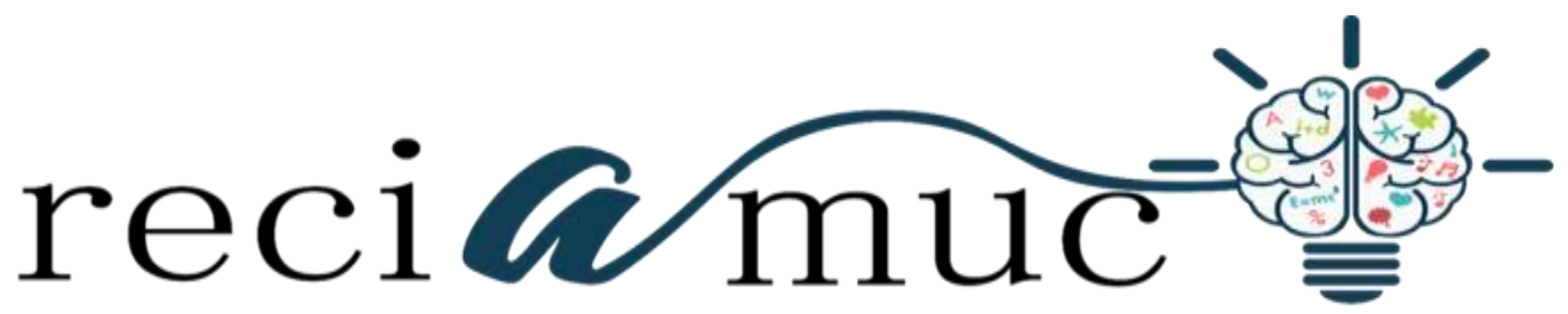

Revista científica de investigación actualización del mundo de las ciencias

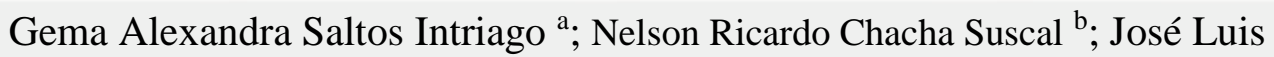

Campoverde Campoverde ${ }^{\mathrm{c}}$; Marjorie Lisbeth Cedeño Velásquez ${ }^{\mathrm{d}}$; Emma Marisela

Mesías Mercado ${ }^{\mathrm{e}}$; Diana Janneth Sánchez Chicaiza ${ }^{\mathrm{f}}$

Procedimientos adecuados para los pacientes en cirugías de corazón abierto

Appropriate procedures for patients in open heart surgeries

Revista Científica de Investigación actualización del mundo de las Ciencias. Vol.

3 núm., 3., julio, ISSN: 2588-0748, 2018, pp. 1156-1175

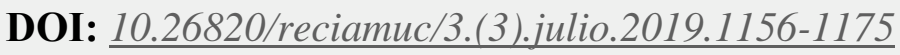

URL: http://reciamuc.com/index.php/RECIAMUC/article/view/322

Código UNESCO: 3205 Medicina Interna

Tipo de Investigación: Artículo de Revisión

(C) RECIAMUC; Editorial Saberes del Conocimiento, 2019

Recibido: 28/04/2019

Aceptado: 19/05/2019

Publicado: 01/07/2019

Correspondencia: gemmys_12@hotmail.com

a. Médico General; Neurorehabilitación Centro Integral; gemmys_12@ hotmail.com; Portoviejo, Ecuador.

b. Médico General; Clínica Cliesmetrosa; ricardocsmed93@gmail.com; La Troncal, Ecuador.

c. Médico General; Clínica Cliesmetrosa; josecampoverde2@gmail.com; La Troncal, Ecuador.

d. Médico Residente; Centro de Salud Santa Ana 24h; wwwdiemar@ hotmail.com; Santa Ana, Ecuador.

e. Médica Cirujana; Hospital de Especialidades Portoviejo; md.emmamesias @ hotmail.com; Portoviejo, Ecuador.

f. Médico Residente; Hospitalización Pediatría - Hospital IESS Quito Sur; dianys-71989@ @otmail.com; Quito, Ecuador. 


\section{Procedimientos adecuados para los pacientes en cirugías de corazón abierto}

vol. 3, núm. 3., (2019)

Gema Alexandra Saltos Intriago; Nelson Ricardo Chacha Suscal; José Luis Campoverde Campoverde; Marjorie Lisbeth Cedeño Velásquez; Emma Marisela Mesías Mercado; Diana Janneth Sánchez Chicaiza

\section{RESUMEN}

Cada día aumentan las enfermedades cardiovasculares manteniéndose como la principal causa de muerte en el mundo. En tal sentido, muchos de los tratamientos de estas enfermedades requieren una cirugía cardíaca, por tanto, son sumamente importantes los procedimientos perioperatorios para alcanzar el éxito de la intervención. El objetivo de este estudio es plasmar los procedimientos postoperatorios adecuados para los pacientes de cirugías a corazón abierto. Para su desarrollo se llevó a cabo una recopilación y revisión de material documental bibliográfico. Existen dos fases de atención postoperatoria, una que se desarrolla en el hospital y posteriormente, la otra en el hogar. Es importante resaltar que, a pesar de la importancia de la unificación de criterios en los procedimientos postoperatorios más adecuados para la recuperación, dichos cuidados deben ser específicos para cada paciente, obedeciendo a una serie de factores que son diferentes de un individuo a otro. Lo imperioso es que el cirujano lidere el equipo que ayuda en la recuperación del paciente y que esté al tanto de del curso y la evolución del mismo, buscando la recuperación física y emocional, de forma que se logre alcanzar la mejor calidad de vida para el paciente, posterior a la operación.

Palabras Claves: Procedimientos; Adecuados; Paciente; Cirugía; Corazón. 


\section{Procedimientos adecuados para los pacientes en cirugías de corazón abierto}

vol. 3, núm. 3., (2019)

Gema Alexandra Saltos Intriago; Nelson Ricardo Chacha Suscal; José Luis Campoverde Campoverde; Marjorie Lisbeth Cedeño Velásquez; Emma Marisela Mesías Mercado; Diana Janneth Sánchez Chicaiza

\section{ABSTRACT}

Cardiovascular diseases increase every day and remain the leading cause of death in the world. In this sense, many of the treatments of these diseases require cardiac surgery, therefore, perioperative procedures are very important to achieve the success of the intervention. The objective of this study is to capture the appropriate postoperative procedures for open heart surgery patients. For its development, a compilation and review of bibliographic documentary material was carried out. There are two phases of postoperative care, one that develops in the hospital and later, the other in the home. It is important to highlight that, despite the importance of the unification of criteria in the most appropriate postoperative procedures for recovery, such care must be specific for each patient, obeying a series of factors that are different from one individual to another. The imperative thing is that the surgeon leads the team that helps in the recovery of the patient and is aware of the course and evolution of the patient, seeking physical and emotional recovery, so that the best quality of life for the patient is achieved. patient, after the operation.

Key Words: Procedures; Suitable; Patient; Surgery; Heart. 


\section{Procedimientos adecuados para los pacientes en cirugías de corazón abierto}

vol. 3, núm. 3., (2019)

Gema Alexandra Saltos Intriago; Nelson Ricardo Chacha Suscal; José Luis Campoverde Campoverde; Marjorie Lisbeth Cedeño Velásquez; Emma Marisela Mesías Mercado; Diana Janneth Sánchez Chicaiza

\section{Introducción.}

Las Enfermedades Cardiovasculares (ECV) constituyen la principal causa de muerte a nivel mundial, superando cada año a cualquier otra causa de muerte. Cálculos indican que para el año 2015 murieron 17,7 millones de personas por esta causa, lo que representa el $31 \%$ del total de muertes registradas en todo el mundo, de las cuales 7,4 millones fueron causadas por cardiopatía coronaria y 6,7 millones a accidentes cerebrovasculares. (Organización Mundial de la Salud, 2017)

Una vez diagnosticado con una enfermedad del corazón, tal como “una enfermedad de las arterias coronarias, una enfermedad de la válvula aórtica, una enfermedad de la válvula mitral, o una enfermedad de la válvula tricúspide”..., existe una gran probabilidad de que el paciente deba reunirse con un cirujano cardiotorácico para evaluar sus opciones de tratamiento. (Sociedad de Cirujanos Torácicos, 2017)

Stoicea, y otros (2017) hacen referencia en su estudio a que en ocasiones la enfermedad cardiovascular y la cardiopatía coronaria pueden llegar a progresar a un punto donde la única opción de tratamiento está constituida por la cirugía coronaria. Citan a la Encuesta De Alta Del Hospital Nacional de los CDC/NCHS (2010) según la cual para ese año hubo 3.891 millones de procedimientos cardíacos. Asimismo, refieren datos del Centro Nacional de Estadísticas de Salud de los Estados Unidos (2015), según el cual, en el año 2013 se realizaron 410.610 cirugías cardíaca en pacientes mayores de 65 años, que representó un 13,0 \% de las altas hospitalarias. (p. 2)

En este sentido, cabe destacar que la cirugía de corazón “es cualquier cirugía realizada en el miocardio, las válvulas, las arterias o la aorta y otras arterias mayores conectadas al corazón"; 


\section{Procedimientos adecuados para los pacientes en cirugías de corazón abierto}

vol. 3, núm. 3., (2019)

Gema Alexandra Saltos Intriago; Nelson Ricardo Chacha Suscal; José Luis Campoverde Campoverde; Marjorie Lisbeth Cedeño Velásquez; Emma Marisela Mesías Mercado; Diana Janneth Sánchez Chicaiza

mientras que el término: cirugía a corazón abierto, se refiere a que el paciente durante la intervención está conectado a una máquina de derivación cardiopulmonar o bien, a una bomba de derivación. Entre los tipos de esta cirugía tenemos: la cirugía de revascularización cardíaca, la cirugía de válvulas cardíacas y la cirugía para corregir el defecto cardíaco presente en el nacimiento. (Biblioteca Nacional de Medicina de los Estados Unidos, 2018)

Posterior a la cirugía a corazón abierto el paciente requerirá de una serie de atenciones y cuidados tanto intrahospitalariamente como en el hogar, y que de ellos dependerá su recuperación, el éxito de la operación e incluso evitar recaídas y posibles nuevas intervenciones.

Como consecuencia inmediata de una operación el paciente está sometido a algunos efectos secundarios, como dolor, hinchazón e inflamación. La mayoría son predecibles y esto permite que el cirujano imparta los procedimientos necesarios para la mejor recuperación del paciente. Por otra parte, existen complicaciones, tales como las infecciones, el sangrado excesivo, las reacciones a la anestesia o alguna lesión accidental, que suelen ser imprevisibles. Las afecciones médicas pueden ser un factor de riesgo para las complicaciones en este tipo de intervenciones. (Biblioteca Nacional de Medicina de los Estados Unidos, 2019)

Para Stoicea et al. (2017) la importancia de la transición de la atención después de la cirugía cardíaca irá avanzando en los siguientes años, dado el aumento de la población anciana y de este tipo de intervenciones. Asimismo, citan a la Oficina del Censo de EE. UU., según la cual "la población de más edad se duplicará entre 2010 y 2050, y las personas mayores de 85 años representan el grupo de mayor crecimiento". (p. 1) 


\section{Procedimientos adecuados para los pacientes en cirugías de corazón abierto}

vol. 3, núm. 3., (2019)

Gema Alexandra Saltos Intriago; Nelson Ricardo Chacha Suscal; José Luis Campoverde Campoverde; Marjorie Lisbeth Cedeño Velásquez; Emma Marisela Mesías Mercado; Diana Janneth Sánchez Chicaiza

En este orden de ideas, el objetivo fundamental de la presente investigación es revisar y plasmar los procedimientos postoperatorios en pacientes de cirugía a corazón abierto, a los fines de puntualizar los métodos más recientes implementados en todo el mundo para la recuperación de este tipo de intervención cardíaca, todo ello con base a la revisión sistemática de material actualizado y fidedigno.

\section{Materiales y Métodos.}

Computadores personales con conexión a internet y el conjunto de una diversa literatura científica dispuesta de manera física, es lo que se concibe para este trabajo de investigación como herramientas y materiales de trabajo, con los que se persigue exponer sobre los procedimientos postoperatorios en pacientes de cirugía a corazón abierto, por lo cual, se propone un diseño de investigación documental o bibliográfica, es decir, un trabajo de tipo no experimental, a nivel descriptivo, de corte transeccional, y bajo una la metodología inductiva.

Esta investigación se limita a la búsqueda y revisión sistemática de material bibliográfico que, por una parte, es accesible mediante el uso de diversas bases de datos, entre las que figuraron: MedlinePlus, PubMed, Biblioteca Virtual de la Salud (BVS), SciELO, Dialnet y ELSEVIER, entre otras; y por la otra, mediante el uso del material físicamente disponible en la biblioteca universitaria, representando todo esto parte esencial del proceso investigativo y de comprensión que facilita la síntesis de la mejor evidencia disponible, y a su vez representando todo ello uno de los resultados esperados.

Principalmente se realizó una búsqueda aleatoria y consecutiva en las mencionadas bases 


\section{Procedimientos adecuados para los pacientes en cirugías de corazón abierto}

vol. 3, núm. 3., (2019)

Gema Alexandra Saltos Intriago; Nelson Ricardo Chacha Suscal; José Luis Campoverde Campoverde; Marjorie Lisbeth Cedeño Velásquez; Emma Marisela Mesías Mercado; Diana Janneth Sánchez Chicaiza

de datos, usando las expresiones "procedimientos postoperatorios”, "cirugía a corazón abierto"; “cuidados postoperatorios" y "cirugía cardiovascular" y "rehabilitación cardíaca", lo que aproximadamente resultó en más de un centenar de miles de registros bibliográficos que luego se fueron filtrando bajo los criterios de idioma español, relevancia, correlación temática y fecha de publicación en los últimos siete años, sin descartar por tipo de material bibliográfico, lo que significa que, se fueron escogiendo títulos de artículos científicos, ensayos, revisiones sistemáticas, protocolos, editoriales, libros, boletines, folletos, tesis de grado, posgrado y doctorado, noticias científicas, entre otros tipos de documentos e información de interés científico y académico.

Seguidamente, se efectúa la selección y clasificación de la literatura científico académica físicamente disponible, aplicando, en términos generales, los mismos criterios que se consideraron para la fuentes digitales, siendo a partir de allí que el equipo investigador le da la correspondiente lectura crítica y análisis de toda esa evidencia científica, lo que resultó consecutiva y consensuadamente en el contenido del presente trabajo, es decir, en el fundamento de las ideas y planteamientos aquí plasmados.

\section{Resultados.}

Procedimientos postoperatorios para pacientes de cirugía a corazón abierto: Fase I. En el hospital

Una vez practicada la cirugía a corazón abierto, el paciente es transferido a la sala de terapia intensiva o Unidad de Cuidados Intensivos (UCI), donde por lo general, tendrá colocado un tubo para ayudarlo a respirar. Estará bajo el efecto de la anestesia, de manera que despertará en la UCI 


\section{Procedimientos adecuados para los pacientes en cirugías de corazón abierto}

vol. 3, núm. 3., (2019)

Gema Alexandra Saltos Intriago; Nelson Ricardo Chacha Suscal; José Luis Campoverde Campoverde; Marjorie Lisbeth Cedeño Velásquez; Emma Marisela Mesías Mercado; Diana

Janneth Sánchez Chicaiza

luego de la intervención. En esta sala de cuidados intensivos, le corresponde al personal de enfermería controlar de forma constante sus signos vitales, tubo de drenaje, respiración, resultados de pruebas e intensidad del dolor. Sacaran los medicamentos anestésicos de forma gradual durante las primeras 2 horas, de esa forma para que el paciente al despertar no requiera de la máquina para respirar y pueda hacerlo por sí mismo. (Sociedad de Cirujanos Torácicos, 2017)

\section{Extubación}

Este procedimiento consiste en quitar el tubo para respirar al paciente, cuando este se encuentre despierto y pueda respirar por sí solo. Una vez quitado el tubo, el paciente es controlado cuidadosamente asegurándose de que su respiración sea normal. El tiempo en que el paciente respira normalmente varía con cada caso, pero por lo general el paciente respira con normalidad unas pocas horas luego de la cirugía. "Cada paciente se despierta de la cirugía de corazón por sí mismo, y a veces se pueden esperar algunas variaciones, especialmente si usted tiene enfermedades preexistentes del pulmón o de los riñones, o una función cardíaca disminuida". (Sociedad de Cirujanos Torácicos, 2017)

\section{Primeros Ejercicios (de respiración y movilidad)}

Posterior a la extubación, al paciente se lo incentiva a realizar ejercicios para la respiración. Asimismo, se evalúa el dolor para el reajuste de los medicamentos, en caso de ser necesario. Estos ejercicios consisten en que el paciente debe tomar una almohada firme y contra ella respire profundamente y tosa, repitiéndolo frecuentemente después de la cirugía, ya que los pulmones se mantuvieron inactivos mientras permanecía conectado a una máquina corazón-pulmón en el 


\section{Procedimientos adecuados para los pacientes en cirugías de corazón abierto}

vol. 3, núm. 3., (2019)

Gema Alexandra Saltos Intriago; Nelson Ricardo Chacha Suscal; José Luis Campoverde Campoverde; Marjorie Lisbeth Cedeño Velásquez; Emma Marisela Mesías Mercado; Diana Janneth Sánchez Chicaiza

transcurso de la intervención. Este ejercicio ayuda a prevenir complicaciones como la neumonía o la acumulación de líquidos alrededor de los pulmones. Con ayuda del personal de enfermería, cuando se sienta listo, el paciente debe salir de la cama y a sentarse en una silla, incluso comenzar a caminar. Es fundamental que se movilice lo antes posible después de la cirugía, esto ayuda a reducir complicaciones como la neumonía, debilidad muscular y los coágulos sanguíneos en las piernas. (Sociedad de Cirujanos Torácicos, 2017)

Es importante destacar que el ejercicio debe ser practicado luego de la cirugía lo antes posible, incluso los primeros días, mientras aún permanece en el hospital el paciente debe ejercitarse cuanto antes mejor, comenzando gradualmente y de acuerdo a su condición con ejercicios de respiración que le permita expandir sus pulmones y evitar secreciones y otras enfermedades, siempre prestando especial atención a la esternotomía. Cualquier movimiento que incluya la cara anterior del pecho, tales como toser o levantarse de la cama, debe realizarse cruzando los brazos hacia la axila contralateral, en todo caso el médico le indicará la forma correcta de realizar la acción. Asimismo, durante estos primeros días postcirugía, se realizan ejercicios sencillos de los miembros inferiores. Hasta que poco a poco el paciente comenzará a dar los primeros pasos. En ningún caso el paciente podrá realizar ningún ejercicio por su cuenta, es decir, sin supervisión. (Masiá Mondéjar, 2018)

\section{De la Unidad de Cuidados Intensivos a otra sala del hospital}

Por orden del equipo de cirugía, el paciente es trasladado de la UCI a otra sala del hospital donde continuará su proceso de recuperación de la cirugía. El propósito de esta nueva sala es que el paciente obtenga un progreso sostenido hasta el punto de que esté preparado para darle de alta 


\section{Procedimientos adecuados para los pacientes en cirugías de corazón abierto}

vol. 3, núm. 3., (2019)

Gema Alexandra Saltos Intriago; Nelson Ricardo Chacha Suscal; José Luis Campoverde Campoverde; Marjorie Lisbeth Cedeño Velásquez; Emma Marisela Mesías Mercado; Diana Janneth Sánchez Chicaiza

y continuar los procedimientos postoperatorios en el hogar. Esta recuperación hospitalaria incluye análisis de laboratorio a diario, así como radiografías del pecho, ejercicios como caminatas por los pasillos, extubar los drenajes, controlar signos vitales, glucosa y respiración. "Se le quitará el catéter urinario para que pueda orinar por su cuenta, pero podría sufrir de estreñimiento (dificultad para defecar) luego de la cirugía”. (Sociedad de Cirujanos Torácicos, 2017)

Procedimientos postoperatorios para pacientes de cirugía a corazón abierto: Fase II. En casa

El cirujano con su equipo son los encargados de tomar las decisiones perioperatorias en las cirugías de corazón abierto, en esta fase, ellos determinarán en qué momento podrá el paciente ser dado de alta del hospital para continuar sus cuidados postoperatorios desde su hogar.

Reglas básicas a seguir en casa luego de la cirugía de corazón

Existen una serie de restricciones o consideraciones para los pacientes operados a corazón abierto, las cuales incluyen: no manejar vehículos durante un mes, no levantar peso, tampoco empujar ni tirar de más de 10 libras, esto por lo menos durante seis semanas. Al bañarse el paciente no debe friccionar las heridas y después del baño debe secarlas muy bien. No debe sumergirse en bañera, piscina, o un jacuzzi hasta haberse curado totalmente sus heridas. El paciente debe estar pendiente en el hogar de la evolución de su herida, y cualquier cambio en ella como el enrojecimiento, que la herida supure, se hinche o se abra, debe acudir e informar al cirujano. Asimismo, debe cualquier cuadro febril (mayor de 101 grados Fahrenheit), o algún malestar como escalofríos excesivos o transpiración nocturna. (Sociedad de Cirujanos Torácicos, 2017) 


\section{Procedimientos adecuados para los pacientes en cirugías de corazón abierto}

vol. 3, núm. 3., (2019)

Gema Alexandra Saltos Intriago; Nelson Ricardo Chacha Suscal; José Luis Campoverde Campoverde; Marjorie Lisbeth Cedeño Velásquez; Emma Marisela Mesías Mercado; Diana Janneth Sánchez Chicaiza

\section{Programas de Rehabilitación Cardíaca. Prevención Secundaria}

La rehabilitación cardíaca es un programa personalizado integral, que incluye ejercicios y educación para pacientes ambulatorios. Está diseñado para ayudar al paciente cardíaco a mejorar su salud y recuperarse, ya sea de un ataque al corazón, cualquier otra forma de enfermedad cardíaca o incluso, cirugía cardiovascular. (Mayo Clinic, 2017)

La Fundación Española del Corazón la define como el "conjunto de métodos que persiguen devolver a los enfermos el mayor número de posibilidades, tanto físicas como mentales, posterior a un evento cardiaco, permitiendo de esta forma recuperar una vida normal en el ámbito social, profesional y familiar. (Valle Muñóz, 2018)

Este tipo de programa constituye una intervención multidisciplinar y se basa en la evidencia. Se trata de una prevención secundaria, que trata de que el paciente cardíaco y/o operado del corazón se recupere y no vuelva a reincidir en el episodio o la cirugía. (Sociedad Española de Cardiología, 2019)

La rehabilitación cardíaca es llevada a cabo a través de un hospital o centro de salud, cuyo propósito es beneficiar a los pacientes que ya se encuentran en el hogar. Para ingresar al programa el paciente requerirá una prescripción a los fines de ser admitido y el tiempo de espera luego de la cirugía para empezar este programa puede variar de cuatro y hasta seis semanas posterior a dicha intervención quirúrgica. (Sociedad de Cirujanos Torácicos, 2017)

\section{Objetivos}




\section{Procedimientos adecuados para los pacientes en cirugías de corazón abierto}

vol. 3, núm. 3., (2019)

Gema Alexandra Saltos Intriago; Nelson Ricardo Chacha Suscal; José Luis Campoverde Campoverde; Marjorie Lisbeth Cedeño Velásquez; Emma Marisela Mesías Mercado; Diana Janneth Sánchez Chicaiza

Los objetivos de la rehabilitación cardíaca son múltiples entre ellos se pueden citar que facilitan el control de los factores de riesgo, mejoran la capacidad física, evitan alteraciones psicológicas como estrés, conocimiento y aceptación del problema cardíaco y se ayuda al paciente a planificar la nueva situación socio-laboral que se le presentará consecuencia de la enfermedad o la cirugía, entre otras. Este programa busca normalizar las relaciones socio-familiares y sexuales del paciente, así como facilitar la reincorporación a nivel laboral, en su conjunto busca mejorar la calidad de vida del paciente. Además, estabiliza la enfermedad arteriosclerótica y retrasar su progresión, disminuye futuros eventos coronarios adversos y por ende, los ingresos por este motivo. Asimismo, disminuye el número de otros procedimientos invasivos y reducir la mortalidad. (Valle Muñóz, 2018)

“Las personas que participan en la rehabilitación cardíaca son menos proclives a reingresar al hospital y alcanzan tasas de supervivencia 25 a 45 por ciento mejores que las personas que no participan en ella”. (Andrade, 2014)

\section{Equipo}

La rehabilitación cardíaca debe estar en manos de un equipo multidisciplinario, profesionales médicos de varias especialidades: expertos en cardiología, enfermería, medicina física y rehabilitación, fisiología del ejercicio, nutrición, endocrinología y medicina del sueño, psicología y psiquiatría, entre otras especialidades. Este equipo es el encargado de diseñar un plan personalizado para las necesidades de cada paciente, supervisan su progreso e informa al proveedor principal de atención médica del paciente. (Andrade, 2014). 


\section{Procedimientos adecuados para los pacientes en cirugías de corazón abierto}

vol. 3, núm. 3., (2019)

Gema Alexandra Saltos Intriago; Nelson Ricardo Chacha Suscal; José Luis Campoverde Campoverde; Marjorie Lisbeth Cedeño Velásquez; Emma Marisela Mesías Mercado; Diana Janneth Sánchez Chicaiza

\section{Medidas}

Son las actividades diseñadas de forma personalizada para cada paciente integradas en la rehabilitación cardiaca, cuyo propósito es mejorar la capacidad funcional del cardiópata. Las medidas básicas de cada programa incluyen: ejercicio físico, control de factores de riesgo, programa psicológico, programa educativo y orientación sociolaboral. (Valle Muñóz, 2018)

\section{Ejercicio Físico}

Constituye una medida clave en la rehabilitación cardíaca, consiste en entrenar al paciente para que haga ejercicio. "En base a la información recogida durante una prueba de ejercicio graduada, el equipo de la rehabilitación cardíaca desarrolla para cada paciente un programa personalizado de ejercicios, conocido como: ejercicios recetados”. (Andrade, 2014)

\section{Control de factores de riesgo}

Esta medida está dirigida a que los participantes aprendan a identificar y mejorar los factores de riesgo cardíaco: la depresión y apnea del sueño, los lípidos sanguíneos, la presión arterial, la glucosa sanguínea o cualquier otro síntoma crónico tales como el dolor del pecho o el cansancio. (Andrade, 2014)

Para Suárez, Barragán y Álvarez, (2015) un programa formal de rehabilitación cardíaca debe promover activamente el cese del tabaquismo, el cual se encuentra asociado con una disminución significativa en la mortalidad, después de un infarto al miocardio. (p. 46)

"El equipo de la rehabilitación cardíaca enseña los principios de una alimentación sana". 


\section{Procedimientos adecuados para los pacientes en cirugías de corazón abierto}

vol. 3, núm. 3., (2019)

Gema Alexandra Saltos Intriago; Nelson Ricardo Chacha Suscal; José Luis Campoverde Campoverde; Marjorie Lisbeth Cedeño Velásquez; Emma Marisela Mesías Mercado; Diana Janneth Sánchez Chicaiza

(Andrade, 2014)

En todo caso el control de los factores de riesgo de la enfermedad cardíaca después de una cirugía a corazón abierto, coadyuva con la recuperación del paciente y evita que este vuelva a recaer y requiera de una nueva intervención, además de mejorar significativamente su calidad de vida.

Programa Psicológico

Es bastante frecuente que los pacientes se sientan deprimidos o tristes después de una cirugía de corazón. La cirugía de corazón es responsable de efectos emocionales en la mayoría de los pacientes. Estos efectos pueden presentarse como tristeza, sentirse cansado, miedo de complicaciones, falta energía, poco apetito, incluso incapacidad para dormir. (Sociedad de Cirujanos Torácicos, 2017)

Cano, Alguacil, Alonso, Molero, \& Miangolarra, (2012) el apoyo psicológico que puedan recibir estos pacientes representa uno de los pilares fundamentales de la rehabilitación cardíaca. (p. 74)

Programa educativo

En la rehabilitación cardíaca se requiere aconsejar y educar al paciente. En esta etapa, los pacientes reciben instrucción sobre su enfermedad, además de la forma en que ellos pueden colaborar para controlar su afección. Asimismo, se educa al paciente acerca de la importancia de tomar los medicamentos según las instrucciones. (Andrade, 2014) 


\section{Procedimientos adecuados para los pacientes en cirugías de corazón abierto}

vol. 3, núm. 3., (2019)

Gema Alexandra Saltos Intriago; Nelson Ricardo Chacha Suscal; José Luis Campoverde Campoverde; Marjorie Lisbeth Cedeño Velásquez; Emma Marisela Mesías Mercado; Diana Janneth Sánchez Chicaiza

\section{Orientación sociolaboral}

Uno de los propósitos básicos de este programa es que el paciente cardíaco, que sufrió un ataque o que fue operado, vuelva a retomar una vida, lo más normalmente posible, según su estado. Que vuelva a la normalidad en su vida cotidiana, en el hogar, con su pareja, amigos y familiares. Incluso, este equipo valorará cuando el paciente se encuentre listo para reingresar a su campo laboral y las nuevas medidas o recomendaciones (en caso de ser necesarias) que este debe adoptar para desarrollar su trabajo y cuidar su salud.

Pérez, Tallón, \& González, (2014) demostraron mediante su estudio que los pacientes que siguen un programa de rehabilitación cardiaca, consiguieron una mejoría de su funcionalidad cardiaca y sensación de bienestar físico y calidad de vida, en tal sentido, consiguieron la indicación a la reincorporación laboral. (p. 378)

El uso de la tecnología en la rehabilitación cardíaca

Las aplicaciones para teléfonos inteligentes se encuentran a la vanguardia del mundo moderno, estos avances también son empleados en la rehabilitación cardíaca de manera satisfactoria. Constituyen una herramienta valiosa que permite al paciente colaborar activamente en el programa y mejorar los resultados mediante el uso de la tecnología.

En un estudio realizado por Mayo Clinic en 44 pacientes, hospitalizados después de un ataque cardíaco se comprobó que el riesgo de reincidir en un evento cardíaco y una nueva hospitalización por tal motivo, se redujo aún más, con el uso de una aplicación para teléfonos inteligentes, además de la rehabilitación cardíaca común. Esta aplicación ayuda al paciente a 


\section{Procedimientos adecuados para los pacientes en cirugías de corazón abierto}

vol. 3, núm. 3., (2019)

Gema Alexandra Saltos Intriago; Nelson Ricardo Chacha Suscal; José Luis Campoverde Campoverde; Marjorie Lisbeth Cedeño Velásquez; Emma Marisela Mesías Mercado; Diana Janneth Sánchez Chicaiza

"registrar la presión sanguínea, el peso, los niveles de la glucosa sanguínea, los minutos de actividad física y los hábitos alimenticios durante un período de tres meses, la aplicación también ofreció a los pacientes actividades educativas para enseñarles otras conductas" ..., ... con la finalidad de evitar futuros problemas cardíacos. (Andrade, 2014)

\section{Rehabilitación Cardíaca Domiciliaria}

Las sociedades científicas americanas: la American Association of Cardiovascular and Pulmonary Rehabilitation (AACVPR), la American Heart Association (AHA) y el American College of Cardiology (ACC), con relación a la rehabilitación cardíaca domiciliaria, "tratan de identificar los componentes básicos, la eficacia, la seguridad y las limitaciones, así como la investigación necesaria para una mejor implantación”. Estos programas domiciliarios representan una buena opción para aquellos pacientes de bajo riesgo o riesgo moderado, que no pueden acudir a los programas impartidos en los hospitales y/o centros de salud. (Sociedad Española de Cardiología, 2019)

\section{Conclusiones.}

La atención del paciente cardíaco en la etapa postoperatoria en fundamental para obtener el éxito de la cirugía practicada. Los procedimientos o cuidados llevados a cabo después de la operación van a influir directamente en la recuperación del paciente, evitan complicaciones, evitan la aparición de otras enfermedades, mejoran el estado general del paciente, evitan la reinserción o práctica de nuevas cirugías, promoviendo una mejor calidad de vida para el paciente operado y la reinserción a su vida normal. 


\section{Procedimientos adecuados para los pacientes en cirugías de corazón abierto}

vol. 3, núm. 3., (2019)

Gema Alexandra Saltos Intriago; Nelson Ricardo Chacha Suscal; José Luis Campoverde Campoverde; Marjorie Lisbeth Cedeño Velásquez; Emma Marisela Mesías Mercado; Diana Janneth Sánchez Chicaiza

Estos procedimientos adecuados o cuidados postoperatorios varían de una región a otra en todo el mundo, no obstante, cada región, país o institución debería poseer un manual para la unificación de los criterios, respaldados en evidencia científica, con la finalidad de obtener los mejores resultados y el mayor bienestar para el paciente de cirugía cardiovascular.

La particularidad común de estos cuidados es que son personalizados, es decir, cada paciente presenta una individualidad que hace que su tratamiento sea único y especial para él, a pesar de que se siga un estándar quien marcará la pauta del proceso será la condición que presente el paciente en las diferentes etapas de su recuperación.

El papel de la rehabilitación cardíaca es fundamental no sólo para tratar al paciente enfermo que ha sufrido un evento cardíaco, sino que representa la garantía de obtener la máxima mejoría de la salud del paciente operado. A pesar de que la inclusión a un programa de este tipo por parte del paciente operado a corazón abierto, pueda tardar con respecto a un paciente que fue hospitalizado por cualquier otro evento relacionado con su enfermedad cardíaca, es importante que cuando su médico lo prescriba, éste ingrese a este tipo de programa, ya que representa la atención integral que necesita y de ello dependerá que vuelva a tener una vida lo más normal posible dentro de su condición y evite requerir nuevas intervenciones a futuro.

La atención postoperatoria cada día está cobrando mayor importancia, por cuanto el número de cirugías mayores como las de corazón abierto aumenta proporcionalmente a las tasas de enfermedades cardiovasculares, en razón del envejecimiento de la población mundial además de otros factores que propician estas enfermedades y que también aumentan más cada día. 


\section{Procedimientos adecuados para los pacientes en cirugías de corazón abierto}

vol. 3, núm. 3., (2019)

Gema Alexandra Saltos Intriago; Nelson Ricardo Chacha Suscal; José Luis Campoverde Campoverde; Marjorie Lisbeth Cedeño Velásquez; Emma Marisela Mesías Mercado; Diana

Janneth Sánchez Chicaiza

\section{Bibliografía.}

Andrade, S. (07 de Mayo de 2014). mayoclinic.org. Recuperado el 26 de Julio de 2019, de https://newsnetwork.mayoclinic.org/discussion/la-rehabilitacion-cardiaca-es-provechosapara-varios-trastornos-del-corazon/

Andrade, S. (15 de Abril de 2014). mayoclinic.org. (M. Clinic, Editor) Recuperado el 26 de Julio de 2019, de https://newsnetwork.mayoclinic.org/discussion/estudio-de-mayo-muestraque-los-pacientes-de-rehabilitacion-cardiaca-que-usan-una-aplicacion-para-telefonosinteligentes-se-recuperan-mejor/

Biblioteca Nacional de Medicina de los Estados Unidos. (28 de Febrero de 2018). MedlinePlus. $\begin{array}{llllll}\text { Recuperado } & \text { el Julio de } 2019, \quad \text { de }\end{array}$ https://medlineplus.gov/spanish/ency/article/002950.htm

Biblioteca Nacional de Medicina de los Estados Unidos. (15 de Febrero de 2019). MedlinePlus. Recuperado el 15 de Julio de 2019, de https://medlineplus.gov/spanish/aftersurgery.html

Cano, R., Alguacil, I., Alonso, J., Molero, A., \& Miangolarra, J. (2012). Programas de rehabilitación cardiaca y calidad de vida relacionada con la salud. Situación actual. Revista Española de Cardiología, 65(1), 72-79. Recuperado el 25 de Julio de 2019, de https://www.revespcardiol.org/es-pdf-S0300893211006968

Masiá Mondéjar, D. (01 de Septiembre de 2018). Fundación Española del Corazón. Recuperado el 25 de Julio de 2019, de https://fundaciondelcorazon.com/ejercicio/ejercicio-fisico/3177ejercicio-fisico-tras-una-cirugia-valvular.html

Mayo Clinic. (09 de Agosto de 2017). mayoclinic.org. Recuperado el 26 de Julio de 2019, de https://www.mayoclinic.org/tests-procedures/cardiac-rehabilitation/about/pac20385192?_ga=2.153493880.1580167951.1564154423-55275360.1533347570 


\section{Procedimientos adecuados para los pacientes en cirugías de corazón abierto}

vol. 3, núm. 3., (2019)

Gema Alexandra Saltos Intriago; Nelson Ricardo Chacha Suscal; José Luis Campoverde Campoverde; Marjorie Lisbeth Cedeño Velásquez; Emma Marisela Mesías Mercado; Diana Janneth Sánchez Chicaiza

Organización Mundial de la Salud. (17 de Mayo de 2017). who.int/es. Recuperado el 01 de Julio de 2019, de https://www.who.int/es/news-room/fact-sheets/detail/cardiovascular-diseases(cvds)

Pérez, I., Tallón, R., \& González, M. (Abril - Junio de 2014). Índice de reinserción laboral tras un programa de rehabilitación cardiaca. MEDICINA y SEGURIDAD DEL TRABAJO, 60(235), 370-378. Recuperado el 25 de Julio de 2019, de http://scielo.isciii.es/pdf/mesetra/v60n235/inspeccion2.pdf

Sociedad de Cirujanos Torácicos. (Mayo de 2017). Recuperado el 26 de Julio de 2019, de https://ctsurgerypatients.org/es/pre-y-post-cuidado-operativo/after-heart-surgery

Sociedad de Cirujanos Torácicos. (Mayo de 2017). ctsurgerypatients.org. Recuperado el 25 de Julio de 2019, de https://ctsurgerypatients.org/es/pre-y-post-cuidado-operativo/beforeheart-surgery

Sociedad Española de Cardiología. (29 de Mayo de 2019). secardiologia. Recuperado el 25 de Julio de 2019, de https://secardiologia.es/blog/10588-documento-de-posicionamiento-deaacvpr-aha-acc-sobre-rehabilitacion-cardiaca-domiciliaria

Stoicea, N., You, T., Eiterman, A., Hartwell, C., Dávila, V., Marjoribanks, S., . . . Rogers, B. (2017). Perspectivas de la transición post-aguda de la atención para pacientes de cirugía cardíaca. Frontiers in Cardiovascular Medicine, 4(70), 1-8. Recuperado el 26 de Julio de 2019, de https://www.ncbi.nlm.nih.gov/pmc/articles/PMC5712014/pdf/fcvm-0400070.pdf

SUÁREZ, P. A., BARRAGÁN, E., \& ÁlVAREZ, Ó. (07 de jULIO de 2015). IMPACTO DE LOS PROGRAMAS DE REHABILITACIÓN CARDÍACA SOBRE LOS FACTORES DE RIESGO CARDIOVASCULAR EN PREVENCIÓN SECUNDARIA. Med, 23(2), 4149. Recuperado el 25 de Julio de 2019, de http://www.scielo.org.co/pdf/med/v23n2/v23n2a05.pdf 
Procedimientos adecuados para los pacientes en cirugías de corazón abierto

vol. 3, núm. 3., (2019)

Gema Alexandra Saltos Intriago; Nelson Ricardo Chacha Suscal; José Luis Campoverde Campoverde; Marjorie Lisbeth Cedeño Velásquez; Emma Marisela Mesías Mercado; Diana Janneth Sánchez Chicaiza

Valle Muñóz, A. (01 de Septiembre de 2018). Fundación Española del Corazón. Recuperado el 25 de Julio de 2019, de https://fundaciondelcorazon.com/informacion-parapacientes/tratamientos/rehabilitacion-cardiaca.html

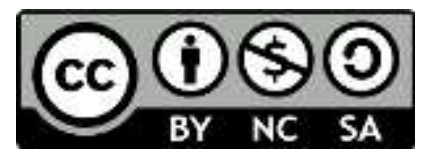

RECONOCIMIENTO-NOCOMERCIAL-COMPARTIRIGUAL

CC BY-NC-SA

ESTA LICENCIA PERMITE A OTROS ENTREMEZCLAR, AJUSTAR Y CONSTRUIR A PARTIR DE SU OBRA CON FINES NO

COMERCIALES, SIEMPRE Y CUANDO LE RECONOZCAN LA AUTORÍA Y SUS NUEVAS CREACIONES ESTÉN BAJO UNA LICENCIA CON LOS MISMOS TÉRMINOS. 